\title{
The Fractal Properties of Retinal Vessels: Embryological and Clinical Implications
}

\author{
MARTIN A. MAINSTER \\ Kansas City, Kansas, USA
}

\begin{abstract}
Summary
The branching patterns of retinal arterial and venous systems have characteristics of a fractal, a geometrical pattern whose parts resemble the whole. Fluorescein angiogram collages were digitised and analysed, demonstrating that retinal arterial and venous patterns have fractal dimensions of $1.63 \pm 0.05$ and $1.71 \pm 0.07$, respectively, consistent with the $1.68 \pm 0.05$ dimension of diffusion limited aggregation. This finding prompts speculation that factors controlling retinal angiogenesis may obey Laplace's equation, with fluctuations in the distribution of embryonic cell-free spaces providing the randomness needed for fractal behaviour and for the uniqueness of each individual's retinal vascular pattern. Since fractal dimensions characterise how completely vascular patterns span the retina, they can provide insight into the relationship between vascular patterns and retinal disease. Fractal geometry offers a more accurate description of ocular anatomy and pathology than classical geometry, and provides a new language for posing questions about the complex geometrical patterns that are seen in ophthalmic practice.
\end{abstract}

Inspection and photography of ophthalmoscopic images are vital to modern ophthalmic practice, but analysis of those images remains largely a subjective process. In other disciplines, images are routinely broken into a large number of tiny picture elements, called pixels, in a process known as digitisation. Computers can digitise, enhance and analyse images. For example, computerised image enhancement can restore ophthalmoscopic detail lost due to ocular media haziness. ${ }^{1}$ Computers can also analyse images, measuring structures ${ }^{2}$ or even quantitating spatial information in much the same way that contrast sensitivity testing characterises an individual's visual performance. ${ }^{3}$

Fractal analysis provides a different way of analysing digitised images, one that is particularly useful when they contain branch- ing patterns. ${ }^{4,5}$ Branching patterns are common in nature, occurring in such diverse phenomena as river tributary networks, lightning discharge pathways, and erosion channels in porous media. Retinal arteries and veins have similar branching patterns.

A branching pattern may have two important fractal properties. First, it may be selfsimilar over a limited range of length scales. That is, it may have similar detail (roughness, branching, etc) when inspected at different magnifications, so that small parts of the pattern exhibit the pattern's overall structure. Second, the pattern may decrease in density as it grows in size. Self-similarity and decreasing density are two important characteristics of fractals, shapes whose parts resemble their whole. $^{4-8}$

Because of self-similarity, fractal properties

Correspondence to: Martin A. Mainster, M.D., Ph.D., Department of Ophthalmology, Kansas University Medical Center, 39th and Rainbow Boulevard, Kansas City, Kansas 66103, USA. 
differ from those of the simple lines and curves of ordinary Euclidean geometry. Since magnifying a fractal only reveals more detail, length is not a useful parameter for describing a fractal. For example, a straight line has the same length regardless of the size of the scale used to measure it. Fractals exhibit more detail at higher magnification, however, so their length increases as the size of the measuring scale decreases. A coastline is a simple example of this principle. ${ }^{4,5}$ An ant crawling along the convoluted edge of a shoreline travels a much greater distance between two locations than an automobile driving between the locations.

Although length is not a useful measure of a fractal, other parameters such as the fractal dimension.(D) are valuable descriptors. The fractal dimension describes a structure's convolutedness. Unlike ordinary Euclidean dimensions that are integers, fractal dimensions are fractions. For example, the Euclidean dimension (E) of a straight line, an area (e.g. the retinal surface), and a volume (e.g. the vitreous gel) are 1,2, and 3, respectively. An irregular but relatively smooth curve has a fractal dimension near one (e.g. 1.2), whilst a highly tortuous curve has a fractal dimension near two (e.g. 1.8).

In a practical sense, a pattern's fractal dimension describes how thoroughly it fills space. For example, squares or circles can completely cover a 2-dimensional space (surface), and their fractal dimension is two. Branching linear structures span 2-dimensional spaces less completely and their fractal dimension is less than two. As a 2-dimensional pattern's fractal dimension increases toward two, the structure covers its surface more completely, and its density drops off more slowly as the pattern increases in size. Decreasing fractal density may have a powerlaw dependence, with an exponent D-E, where $D$ is the fractal dimension of the pattern, and $\mathrm{E}$ is the Euclidean dimension of the space containing it.

The diffusion-limited aggregation (DLA) model of Witten and Sander has helped to explain how growth and structure are related in fractal branching patterns. ${ }^{6-9}$ Computergenerated DLA patterns are similar in appearance and fractal dimension to patterns produced by dielectric breakdown,${ }^{10}$ electrodeposition, ${ }^{11}$ viscous fingering, ${ }^{5,12-15}$ and a variety of other physical phenomena. ${ }^{4-8}$ They also resemble human retinal arterial and venous patterns. Since DLA behaviour could offer insight into the relationship between retinal angiogenesis and the geometry of mature retinal vessels, retinal vessel patterns were studied to determine if they have fractal properties and a fractal dimension consistent with DLA.

\section{Methods}

Arterial and venous patterns were identified in the wide-field fluorescein angiogram collages $^{16}$ of six individuals with early background diabetic retinopathy. A map of retinal arteries and veins of each individual was prepared for vessels larger than capillaries. Arteries and veins were digitised separately, with the optic disk centred on a two-dimensional $1000 \times 1000$ array of square pixels. Figures 1-3 show the retinal vessel map and digitised arterial and venous patterns of the left eye of a 57 year old woman. Pixel size on the retina was $20 \mu \mathrm{m}$, assuming an average optic disc diameter of $1.5 \mathrm{~mm}$ in retinal photographs.

Digitising an arterial or venous pattern consists of determining whether each pixel is occupied by any part of the vascular pattern. Each black square or circle in Figure 2 or 3 represents a pixel occupied by part of the arterial or venous pattern, respectively. The remaining pixels were vacant of vascular detail and are not shown.

There are several ways to determine the fractal dimension of a digitised retinal vascular pattern..$^{5-7,17-20}$ The simplest method is to construct a series of circles of different radii centred about the optic nerve, and count the 'mass' (number) of occupied pixels within a circle of a given radius. If the logarithm of the pixel mass within a circle of a given radius is then plotted against the logarithm of the radius of the circle, the slope of the log-log plot is $\mathrm{D}$, the fractal dimension of the pattern. A fractal dimension can also be determined from pixel density, mass/area, where area is altered to account for projection of the curved retina onto a flat ophthalmoscopic image.

Mapping, digitisation and analysis were 


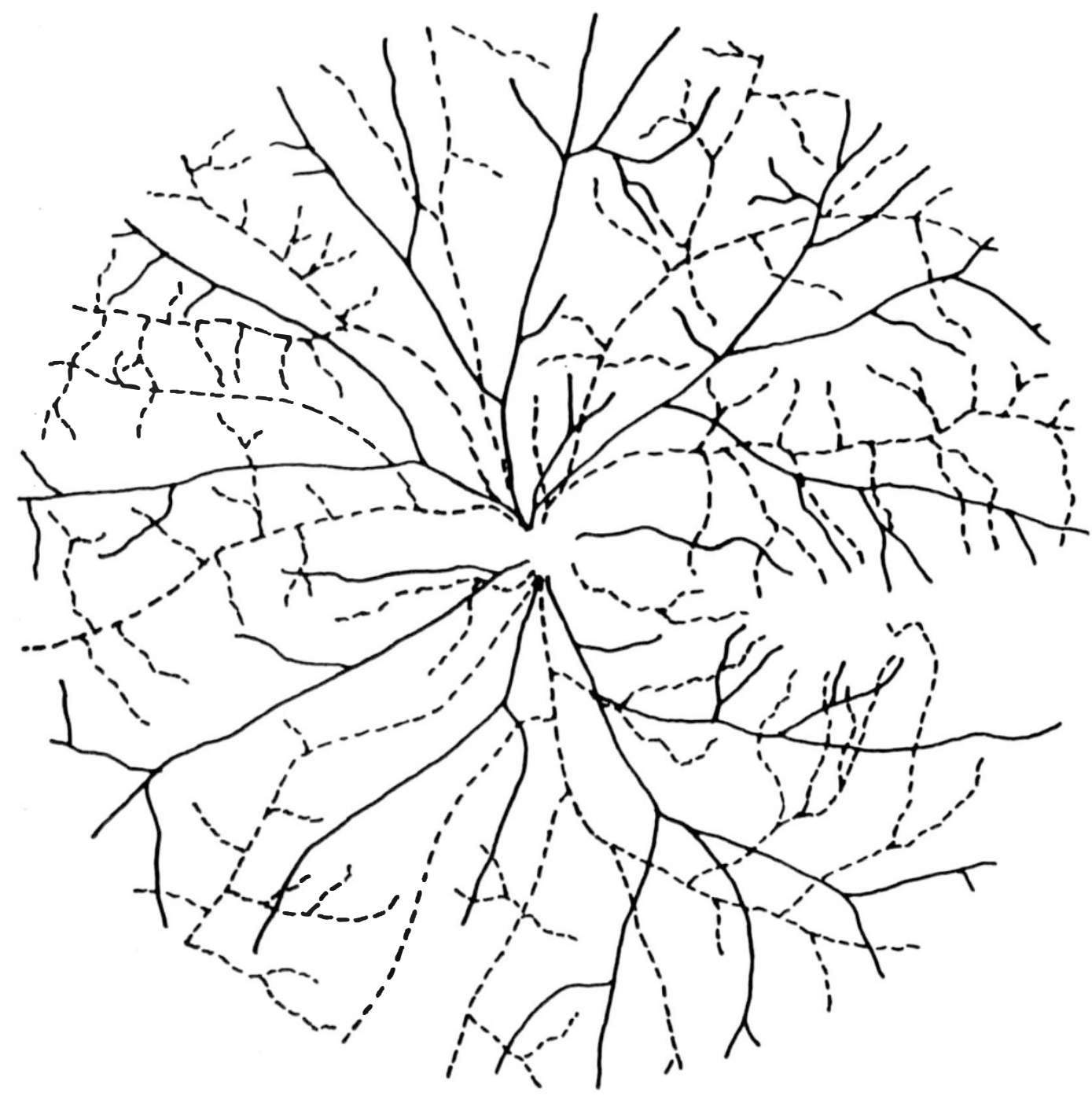

Fig. 1. Retinal arterial (- ) and venous (-- - - ) systems in the fluorescein angiogram collage of the left eye of a 57 year old woman. The optic nerve is in the centre of the branching pattern, with the macula to its right.

carried out manually, and required over 20 hours to analyse a single angiogram. In the future, video retinal photography systems will have the required $1024 \times 1024$ (or, optimally, $2048 \times 2048$ ) pixel resolution needed for this type of analysis. Computer algorithms then could be developed for comparing different angiogram phases to differentiate between arterial and venous systems and for compounding fractal dimensions by methods ${ }^{5-7,17-20}$ such as the mass-radius relationship discussed above.

\section{Results}

Retinal vascular density decreases from the central to peripheral retina, following a power-law relationship with mean arterial and venous fractal dimensions of $1.63 \pm 0.05$ and $1.71 \pm 0.07$, respectively ( \pm std). The difference between arterial and venous fractal dimensions was statistically significant $(P<0.05$, two-tailed t test $)$. Fractal dimensions change by less than $3 \%$ with a correction for retinal curvature estimated from emmetropic model eye parameters. ${ }^{21}$ Fractal dimen- 


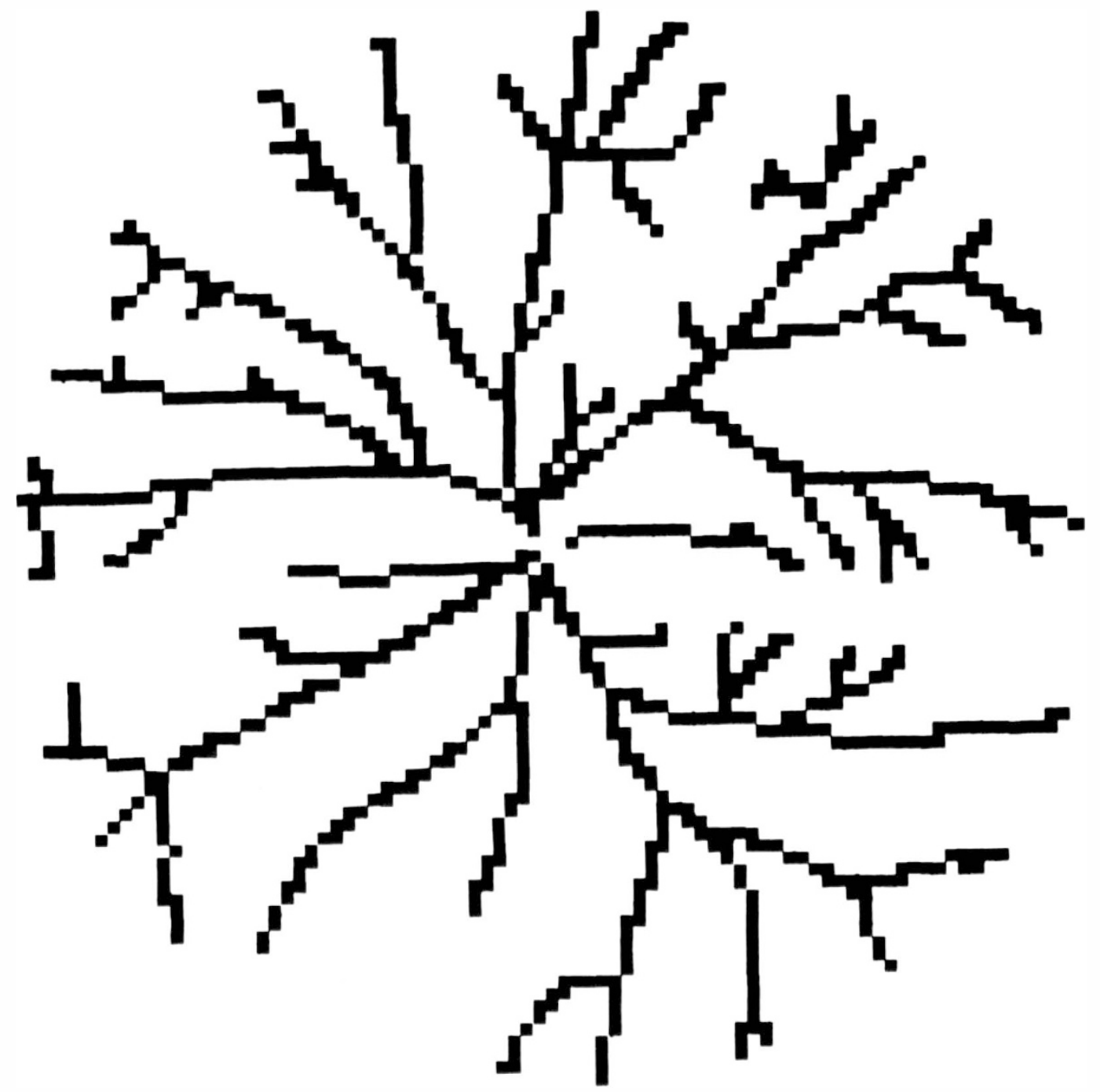

Fig. 2. Retinal arterial pattern for the individual in Fig. 1, digitised on a $1000 \times 1000$ array of square $20 \mu \mathrm{m}$ pixels. The optic nerve is in the centre of the branching pattern, with the macula to its right. Each black square is a pixel occupied by part of the arterial system (squares may be contiguous with adjacent squares). The remaining pixels are vacant of vascular detail and not shown. The fractal dimension for the arterial pattern is 1.61.

sions from fluorescein angiogram analysis should be more sensitive to vascular non-perfusion than narrowing.

It is interesting to note that earlier morphologic data ${ }^{22}$ on retinal capillary density can be re-expressed in a power-law relationship, yielding a fractal dimension of 1.82. Thus, proceeding outward from the optic disc to the peripheral retina, capillary density drops off more slowly than venous density $(\mathrm{D}=1.71)$, which drops off more slowly than arterial density $(D=1.63)$. In other words, the retina is spanned most completely by the capillary system, less completely by the venous system, and least completely by the arterial system.

\section{Discussion}

Retinal arterial and venous patterns have a structure and fractal dimension similar to those of two-dimensional DLA and related phenomena. The fractal dimension of computer-simulated two-dimensional DLA is 


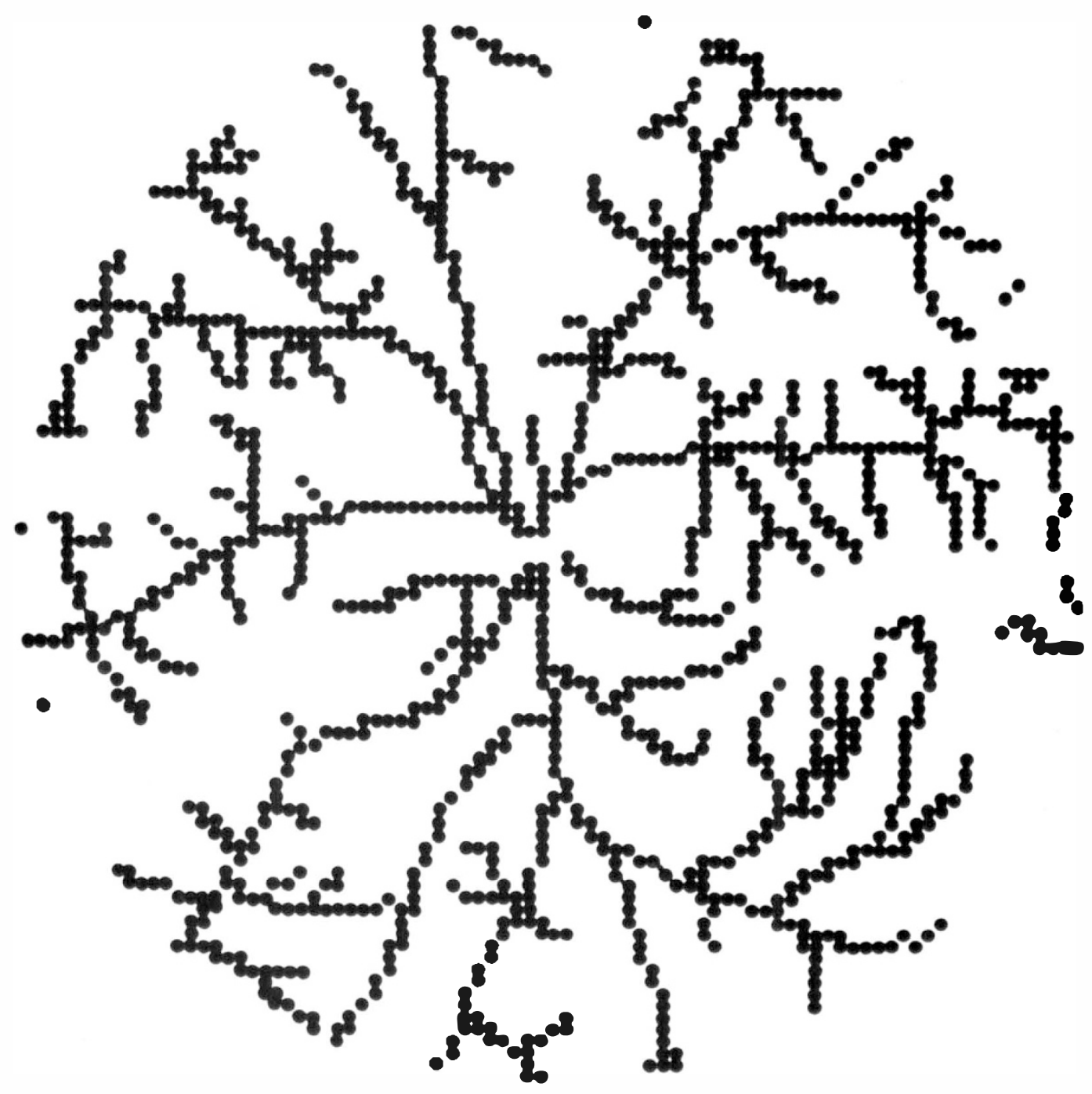

Fig. 3. Retinal venous pattern for the individual in Fig. 1, digitised on a $1000 \times 1000$ array of square 20 um pixels. The optic nerve is in the centre of the branching pattern, with the macula to its right. Each black circle is a pixel occupied by part of the venous system. The remaining pixels are vacant of vascular detail and not shown. The fractal dimension for the venous pattern is 1.76 .

$1.68 \pm 0.05,{ }^{23}$ similar to the fractal dimensions measured for physical phenomena such as dielectric breakdown $(\approx 1.7),{ }^{10}$ electrodeposition $(1.66 \pm 0.03),{ }^{11}$ and viscous fingering $(1.64 \pm 0.04) .{ }^{14}$ The retinal capillary system has a larger fractal dimension than DLA structures.

DLA-like phenomena probably produce similar patterns because their non-equilibrium growth is governed by Laplace's equation and appropriate boundary condi- tions. ${ }^{6-8,12}$ In viscous fingering, for example, threadlike branching fingers develop as water under pressure displaces a more viscous fluid in a porous medium. ${ }^{5,12-15}$ Pressure is governed by Laplace's equation, whilst local fluctuations in pore geometry of the porous material introduce the randomness neededfor fractal behaviour. ${ }^{6-8,12}$ This process has a remarkable parallel in retinal angiogenesis.

Retinal vessels develop from primitive mesenchymal cells. These cells differentiate into 
cords of endothelial cells that canalise to form an interconnecting polygonal (usually pentagonal) capillary network. ${ }^{24-26}$ An annular zone of capillary formation spreads radially outward from the optic disc. Capillary remodelling at the trailing edge of the centrifugal angiogenic wave is directed by haemodynamic factors such as arterial pressure and chemical factors such as oxygen demand and diffusable growth modulators from the retinal pigment epithelium. Capillary hypertrophy and retraction produce the larger arterial and venous blood vessels of the mature retina.

Capillary and larger vessel formation probably occur in inner retinal cell-free spaces separated by Muller cell cytoplasmic extensions. Thus, arterial and venous patterns may represent adaptations within the constraints imposed by the lateral distribution of cell-free spaces. ${ }^{24-26}$ In view of the similarity between vascular patterns and DLA, it is tempting to speculate that Laplace's equation describes the global haemodynamic and metabolic forces responsible for canalisation and expansion of angioblastic cords, whilst local fluctuations in cell-free space geometry provide the randomness needed for fractal geometry and the uniqueness of each individual's retinal vascular pattern.

It should be emphasised that a simple twodimensional DLA model does not account for the absence of vascularisation in the fovea or outer retina. One factor that could limit vascularisation in the macular region is the spatial hindrance of competition for cell-free spaces between developing vessels and ganglion cells. ${ }^{25}$ Although two-dimension DLA is consistent with the lateral branching patterns of the retinal arterial and venous systems, the retinal capillary system's fractal dimension is too large for DLA. Furthermore, a twodimensional model cannot account for axial separation of capillary networks or the absence of outer retinal vascularisation. This vertical vascular stratification emphasises that the determinants of evolving spatial vascular patterns include not only spatial factors, but also hydrostatic, chemical and perhaps even electrical ones as well.

Angiogenesis in the embryonic retina from capillary remodelling is fundamentally different from neovascularisation in the mature ret- ina due to vascular budding. Neovascularisation produces polarised fractal-canopy networks ${ }^{4}$ that have a smaller fractal dimension than normal retinal vessel patterns. Differences between the vascular architecture of human and non-human retinae or between retinal and non-retinal tissues may arise from differences in a variety of parameters such as vascular growth rate; the number and location of arterial sources; local geometrical factors such as retinal thickness and curvature; and the size, distribution and regularity of embryonic spaces available for vascular growth and development.

Laplacian patterns are common in the eye. For example, the retinal nerve fibre layer has the form of lines of force in a two-dimensional Laplacian field (e.g. an electric field), with a point sink (e.g. a negative charge) at the optic nerve, and a point source (e.g. a positive charge) displaced temporally from the fovea. Feynman ascribed the ubiquity of Laplacian processes in nature to the smoothness of spacetime. ${ }^{27}$ The randomness needed for fractal vascular branching in the retina may arise from the graininess of the milieu in which Laplacian forces act during angiogenesis. Demonstration that the retinal vasculature has DLA-like characteristics does not identify the formative Laplacian forces or the origin of local graininess, but it does suggest that these may be key factors in normal retinal angiogenesis and that fractal analysis may offer new insight into retinal vascular development.

Fractal analysis may also offer insight into retinal vascular dysfunction. If differences in how effectively vascular patterns span the retina are significant in determining individual susceptibility to disorders such as diabetic retinopathy, fractal dimension measurements could answer potentially significant clinical questions. For example, are diabetics with retinal vessel patterns of relatively low fractal dimension at increased risk of diabetic retinopathy? Do venous fractal dimensions decrease with progression of diabetic retinopathy, and if so, is this change a harbinger of neovascularisation? Can fractal dimensions help individualise grid laser photocoagulation patterns for treating diabetic macular oedema?

Similar qustions can be posed for other ophthalmic problems. For example, does the 
dendritic pattern of herpes simplex keratitis imply that Laplacian forces play a role in its pathogenesis? Does the dendrite's fractal dimension indicate its virulence? The list of potential questions is lengthy. In general, fractal theory offers a new geometry of nature, one that represents the realities of ocular anatomy and pathology more accurately than the lines and circles of classical geometry. Equally significantly, fractal geometry provides a new language for examining and questioning the complex patterns we view in modern ophthalmic practice.

This research was supported by the Kansas Lions Sight Foundation, Inc. and Research to Prevent Blindness, Inc.

\section{References}

${ }^{1}$ Peli, E and Schwartzß: Enhancement of fundus photographs taken through cataracts. Ophthalmology 1987, 94(S): 10-3.

${ }^{2}$ Ward NP, Tomlinson S, Taylor CJ: Image analysis of fundus photographs: the detection and measurement of exudates associated with diabetic retinopathy. Ophthalmology 1989, 96: 80-6.

${ }^{3}$ Jindra LF, van Saarloos PP, Eikelbloom RH, Cooper RL: Fourier analysis of the nerve fiber layer by digital imaging techniques. Ophthalmic Surg 1989, 20: $370-2$.

${ }^{4}$ Mandelbrot BB: The fractal geometry of nature. New York. WH Freeman, 1983, 151-65.

${ }^{5}$ Feder J: Fractals. New York. Plenum Press, 1988, 6-61.

${ }^{6}$ Witten TA and Cates ME: Tenuous structures from disorderly growth processes. Science 1986, 232: 1607-12.

${ }^{7}$ Sander LM: Fractal growth processes. Nature 1986, 322: 789-93.

${ }^{8}$ Sander LM: Fractal growth. Scientific Am 1987, (Jan), 256: 94-100.

${ }^{9}$ Witten TA Jr and Sander LM: Diffusion limited aggregation, a kinetic critical phenomenon. Phys Rev Lett 1981, 47: 1400-3.

${ }^{10}$ Niemeyer L, Pietronero L, Wiesmann HJ: Fractal dimension of dielectric breakdown. Phys Rev Lett 1984, 52: 1033-6.

${ }^{11}$ Matsushita M, Sano M, Hayakawa Y, Honjo H, Sawada Y: Fractal structures of zinc metal leaves grown by electrodeposition. Phys Rev Lett 1984, 53: 286-9.

${ }^{12}$ Paterson L: Diffusion-limited aggregation and twofluid displacements in porous media. Phys Rev Lett 1984, 52: 1621-4.

${ }^{13}$ Nittmann J, Daccord G, Stanley HE: Fractal growth of viscous fingers: quantitative characterisation of a fluid instability phenomenon. Nature 1985,314 : $141-4$.

${ }^{14}$ Maloy KJ, Boger F, Feder J, Jossang T: Dynamics of viscous-fingering fractals in porous media. Phys Rev A 1987, 36: 318-24.

${ }^{15}$ Oxaal U, Murat M, Boger F, Aharony A, Feder J, Jossang $\mathrm{T}$ : Viscous fingering on percolation clusters. Nature 1987, 329: 32-7.

${ }^{16}$ Niki T, Muraoka K, Shimizu K: Distribution of capillary nonperfusion in early-stage diabetic retinopthy. Ophthalmology 1984, 91: 1431-9.

${ }^{17}$ Forrest SR and Witten TA Jr; Long-range correlations in smoke-particle aggregates. J Phys A 1979, 12: L109-17.

${ }^{18}$ Nittman J, Daccord G, Stanley HE: When are viscous fingers fractal? In, Pietronero L, Tosatti E, Eds. Fractals in Physics, Amsterdam: North-Holland 1986, 193-202.

${ }^{19}$ Dewdney AK: Computer recreations: random walks that lead to fractal crowds. Scientific Am 1988, (Dec), 259: 116-9.

${ }^{20}$ Vicsek T: Fractal growth phenomna. Singapore. World Scientific, 1989, 71-97.

${ }^{21}$ Drasco $\mathrm{N}$ and Fowler CW: Non-linear projection of the retinal image in a wide-angle schematic eye. Br J Ophthalmol 1974, 58: 709-14.

${ }^{22}$ Toussaint D and Danis P: Vascular density coefficients in normal human retina and relationship to distance from disc. Arch Ophthalmol 1970, 83: 281-5.

${ }^{23}$ Meakin P: Diffusion-controlled cluster formation in 2-6 dimensional space. Phys Rev A 1983, 27: 1495-507.

${ }^{24}$ Ashton N: Retinal angiogenesis in the human embryo. Br Med Bull 1970, 26: 103-6.

${ }^{25}$ Flower RW, McLeod DS, Lutty GA, Goldberg B, Wajer SD: Postnatal retinal vascular development of the puppy. Invest Ophthalmol Vis Sci 1985, 26: 957-68.

${ }^{26}$ McLeod DS, Lutty GA, Wajer SD, Flower RW: Visualisation of a developing vasculature. Microvasc Res 1987, 33: 257-69.

${ }^{27}$ Feynman RP, Leighton RB, Sands M: The Feynman lectures on physics, vol.2. Reading. AddisonWesley, 1964, 12-12. 